\title{
artigo
}

Dantas Silva, R.R.D.; Santos, T.S.; Ramos, W.T; Barreiro, M.S.C.; Mendes, R.B.; Freitas, C.K.A.C.

Ações do enfermeiro para prevenção e detecção precoce do câncer de mama

\section{Ações do enfermeiro para prevenção e detecção precoce do câncer de mama}

\author{
Nurse's actions for prevention and early detection of breast cancer \\ Acciones de la enfermera para la prevención y detección precoz del cáncer de mama
}

\begin{abstract}
RESUMO
Objetivo: Identificar as ações realizadas pelo enfermeiro para prevenção e detecção precoce do câncer de mama na Estratégia de Saúde da Família. Método: Trata-se de uma revisão integrativa cuja busca de artigos foi realizada nas bases de dados LILACS, BDENF e SciELO, sendo explorados artigos originais dos últimos cinco anos. Resultados: Foram encontrados 182 artigos. Após a aplicação dos critérios de seleção, obteve-se 10 artigos para análise, sendo predominante os estudos de método transversal com abordagem qualitativa. Percebeu-se que o enfermeiro, através das consultas de enfermagem, exame clínico e solicitação de exames, é fundamental para prevenção e detecção precoce do câncer de mama. Ações educativas também ocorrem, como orientaçães para autoexame e redução dos fatores de risco modificáveis. Conclusão: A atuação do enfermeiro frente à prevenção e detecção do câncer de mama é ampla. Entretanto, é necessário que aja educação permanente desses profissionais, além de protocolos que ampliem sua autonomia.
\end{abstract}

DESCRITORES: Neoplasia da mama; Detecção precoce de câncer; Enfermagem; Atenção Primária à Saúde.

\section{ABSTRACT}

Objective: To identify the actions taken by nurses for the prevention and early detection of breast cancer in the Family Health Strategy. Method: This is an integrative review whose search for articles was carried out in the LILACS, BDENF and SciELO databases, with original articles from the last five years being explored. Results: 182 articles were found. After applying the selection criteria, 10 articles were obtained for analysis, with a predominance of cross-sectional studies with a qualitative approach. It was noticed that the nurses, through nursing consultations, clinical examination and ordering tests, are essentials for prevention and early detection of breast cancer. Educational actions are also done, such as guidelines for self-examination and reduction of modifiable risk factors. Conclusion: The role of nurses in the prevention and detection of breast cancer is broad. However, there is a need for permanent education of these professionals, in addition to protocols that expand their autonomy. DESCRIPTORS: Breast neoplasm. Early detection of cancer. Nursing. Primary Health Care.

\section{RESUMEN}

Objetivo: Identificar las acciones realizadas por los enfermeros para la prevención y detección temprana del cáncer de mama en la Estrategia Salud de la Familia. Método: Se trata de una revisión integradora cuya búsqueda de artículos se realizó en las bases de datos LILACS, BDENF y SciELO, explorando artículos originales de los últimos cinco años. Resultados: Se encontraron 182 artículos. Luego de aplicar los criterios de selección, se obtuvieron 10 artículos para análisis, con predominio de estudios transversales con abordaje cualitativo. Se notó que la enfermera, a través de las consultas de enfermería, el examen clínico y la solicitud de pruebas, es fundamental para la prevención y detección temprana del cáncer de mama. También se llevan a cabo acciones educativas, como pautas de autoexamen y reducción de factores de riesgo modificables. Conclusión: El papel de los enfermeros en la prevención y detección del cáncer de mama es amplio. Sin embargo, existe la necesidad de una formación permanente de estos profesionales, además de protocolos que amplíen su autonomía.

DESCRIPTORES: Neoplasias de mama. Detección precoz de cáncer. Enfermería. Primeros auxilios.

RECEBIDO EM: 30/01/2021 APROVADO EM: 11/02/2021

\section{Renata Roberta Dantas Silva}

Enfermeira. Universidade Paulista, Especialista em emergência e UTI; Especialista em saúde pública com ênfase no ESF; Especialista em docência em enfermagem.

ORCID: 0000-0002-9134-978X 
Thaiane Santana Santos

Graduanda de Enfermagem- UFS. Universidade Federal de Sergipe.

ORCID: 0000-0003-2207-8055

\section{Willamis Tenório Ramos}

Graduando de Fisioterapia- UFS. Universidade Federal de Sergipe.

ORCID: 0000-0002-6037-2856

\section{Maria do Socorro Claudino Barreiro}

Enfermeira. Doutora em Ciências da Saúde, Universidade Federal de Sergipe.

ORCID: 0000-0001-9823-4638

\section{Rosemar Barbosa Mendes}

Enfermeira. Doutora, Universidade Federal de Sergipe.

ORCID: 0000-0002-4860-7413

\section{Carla Kalline Alves Cartaxo Freitas}

Enfermeira. Doutora em Ciências da Saúde, Universidade Federal de Sergipe.

ORCID: 0000-0001-7604-9132

\section{INTRODUÇÃO}

A transição demográfica vem acompanhada de modificações epidemiológicas que alteram o perfil de morbimortalidade da população, com prevalência de doenças crônicas não transmissíveis (DCNT), tais como o câncer que constitui um problema de saúde pública que tem tomado proporções incontroláveis, principalmente nos países em desenvolvimento, sendo considerada a segunda causa de morte na população brasileira ${ }^{1,2}$.

Nesse cenário, as medidas tomadas no Brasil frente ao quadro de saúde do país só se voltaram ao controle do câncer no final do século XX, tendo o seu marco com o estabelecimento do Instituto Nacional de Câncer José Alencar Gomes da Silva (INCA) como órgão assistente do Ministério da Saúde a fim de orientar e coordenar as ações oncológicas, além de realizar pesquisa e educação em saúde na área ${ }^{3}$. Desde então, é de competência do instituto, além de outras atividades assistenciais e de gestão, a participação na formulação da política nacional de prevenção, diagnóstico e tratamento do câncer ${ }^{4}$.

Uma vez compreendido o caráter crônico da doença, o INCA propõe publicações com parâmetros epidemiológicos do câncer a cada três anos desde 2006. Dentre as estimativas publicadas pelo instituto para o triênio 2020-2022 no Brasil,
Assim, a detecção

através de

programas de

rastreamento

representa uma

alternativa

que favorece $o$

diagnóstico

em fases iniciais

da doença, o que

contribui para o

tratamento curativo

e/ou maiores taxas

de sobrevida. aponta-se aproximadamente 625 mil novos casos. Destaca-se, ainda, a incidência do câncer de pele não melanoma com 177 mil casos, seguido dos tumores de próstata e mama (66 mil cada). Atualmente, o câncer de mama é considerado a primeira neoplasia mais incidente entre as mulheres, com risco estimado de 61 casos para cada 100 mil mulheres .

As elevadas taxas de mortalidade são ocasionadas pelo diagnóstico tardio, motivo pela qual tornam o rastreamento e detecção precoce principais medidas de controle da doença. Dentre os métodos mais eficazes de detecção precoce, destacam-se a mamografia (MMG) e o exame clínico das mamas (ECM) que constituem intervenções em todos os níveis de atenção à saúde da mulher ${ }^{6}$.

No contexto da Estratégia de Saúde da Família (ESF), as ações para o controle do câncer de mama são direcionadas a promoção da saúde e prevenção da doença, além do diagnóstico precoce e apoio ao tratamento instituído na atenção secundária e terciária. Assim, a detecção através de programas de rastreamento representa uma alternativa que favorece o diagnóstico em fases iniciais da doença, o que contribui para o tratamento curativo e/ou maiores taxas de sobrevida ${ }^{7}$.

Desse modo, ao analisar a literatura científica, tem se observado poucas produções que enfatizem as ações do enfermeiro 


\section{artigo}

frente ao câncer de mama na ESF, o que evidencia uma lacuna de conhecimento quanto à educação em saúde com ênfase na incidência e nas estratégias de identificação em nível primário, por constituir a porta de entrada para os serviços públicos de saúde ${ }^{8}$. Diante disso, a pesquisa teve como objetivo identificar, nas produções científicas, as principais ações realizadas pelo enfermeiro para prevenção e detecção precoce do câncer de mama na Estratégia de Saúde da Família, além das limitações desse profissional na execução dessas atividades.

\section{MÉTODO}

Trata-se de uma revisão integrativa realizada de acordo com as seguintes etapas propostas por Mendes, Silveira e Galvão9: a) definição da pergunta norteadora; b) busca de seleção dos estudos primários; c) extração de dados dos estudos primários; d) avaliação crítica dos estudos primários; e) síntese dos resultados da revisão; f) apresentação da revisão.

Assim, a revisão foi norteada pela pergunta: Quais são as principais ações realizadas pelo enfermeiro para prevenção e detecção precoce do câncer de mama na ESF descritas na literatura?

Diante disso, realizou-se uma busca nas bases de dados eletrônicas: Base de Dados de Enfermagem (BDENF), Literatura Latino-Americana e do Caribe em Ciências da Saúde (LILACS) e Scientific Electronic Library Online (SciELO). A partir da terminologia em saúde DeCS, da Biblioteca Virtual em Saúde (BVS), foram selecionados os descritores: Neoplasias da Mama; Enfermagem; Detecção Precoce de Câncer e Atenção Primária à Saúde.

A coleta de dados ocorreu durante o mês de fevereiro de 2020 e obedeceu aos seguintes critérios de inclusão: artigos publicados entre o período de 2015 a 2020, em português, disponíveis na íntegra e gratuitamente. Foram excluídos os artigos duplicados, dissertações ou teses.

A seleção da amostra foi realizada em duas etapas. Na primeira, o refinamento ocorreu pela leitura dos títulos dos artigos e dos resumos, sendo excluídos os que não tivessem relação com o tema. Posteriormente, após a identificação, aplicação dos critérios de seleção e leitura, foram selecionados aqueles que se enquadravam perfei- tamente aos critérios de inclusão.

$\mathrm{Na}$ primeira busca foram encontrados 182 artigos. Ao término da busca em todas as bases eletrônicas, foi realizada a leitura e análise dos artigos encontrados, bem como a seleção criteriosa de acordo com os critérios pré-estabelecidos. Com isso, foram excluídos 172 artigos, resultando em 10 que compuseram a amostra.

Para extrair os dados dos estudos selecionados, foi utilizado um instrumento que permitiu avaliar separadamente cada artigo, o qual contemplou os seguintes itens: título, ano, periódico, metodologia e principais resultados encontrados. Por fim, os artigos foram submetidos a um processo de extração de dados e, com base nisso, construído o texto dessa revisão integrativa.

\section{RESULTADOS}

Ao realizar a análise dos 10 artigos, estes foram caracterizados mediante autores e ano, método, periódico, amostra e principais ações realizadas pelo enfermeiro na prevenção e detecção precoce do câncer de mama. Essas informações encontram-se dispostas na tabela 1 .

\section{Tabela 1- Síntese dos artigos incluídos na revisão integrativa (Aracaju/SE, 2020)}

\begin{tabular}{|c|c|c|c|c|c|}
\hline $\mathbf{N}^{0}$ & AUTORES/ ANO & MÉTODO & PERIÓDICO & AMOSTRA & PRINCIPAIS AÇÕES \\
\hline A1 & $\begin{array}{l}\text { DUARTE et al., } \\
2015^{10}\end{array}$ & $\begin{array}{l}\text { Descritivo } \\
\text { exploratório } \\
\text { Quantitativo }\end{array}$ & $\begin{array}{l}\text { Revista de Enferma- } \\
\text { gem do Centro-Oes- } \\
\text { te Mineiro }\end{array}$ & 300 usuárias & $\begin{array}{c}\text { Orientações, exame de mamas, consulta de } \\
\text { enfermagem, educação em saúde. }\end{array}$ \\
\hline$A 2$ & $\begin{array}{l}\text { ZAPPONI; TOCAN- } \\
\text { TINS; VARGES, } \\
2015^{11}\end{array}$ & $\begin{array}{l}\text { Descritivo } \\
\text { Fenomenológico } \\
\text { Qualitativo }\end{array}$ & $\begin{array}{l}\text { Revista de Enferma- } \\
\text { gem da UERJ }\end{array}$ & 12 enfermeiros & $\begin{array}{l}\text { Educação em saúde, exame das mamas, } \\
\text { capacitação das mulheres para AEM, }\end{array}$ \\
\hline A3 & $\begin{array}{l}\text { MORAES et al., } \\
2016^{12}\end{array}$ & $\begin{array}{l}\text { Descritivo } \\
\text { Transversal }\end{array}$ & $\begin{array}{l}\text { Revista de enferma- } \\
\text { gem da USP }\end{array}$ & 60 enfermeiros & $\begin{array}{l}\text { Consulta de enfermagem, ECM, orienta- } \\
\text { ções, busca ativa, ações educativas. }\end{array}$ \\
\hline A4 & $\begin{array}{l}\text { MARQUES; SILVA; } \\
\text { GUTIÉRREZ, } 2017^{13}\end{array}$ & Transversal & $\begin{array}{l}\text { Revista de Enferma- } \\
\text { gem da UERJ }\end{array}$ & 13 enfermeiros & $\begin{array}{l}\text { Palestras, consulta de enfermagem, AEM, } \\
\text { encaminhamento dos casos suspeitos. }\end{array}$ \\
\hline A5 & MELO et al., $2017^{14}$ & Transversal & $\begin{array}{l}\text { Revista Brasileira de } \\
\text { Enfermagem }\end{array}$ & 133 enfermeiros & $\begin{array}{l}\text { ECM, ações educativas, consulta de enfer- } \\
\text { magem, agendamento de retorno e busca } \\
\text { ativa. }\end{array}$ \\
\hline A6 & $\begin{array}{l}\text { TEIXEIRA et al., } \\
2017^{15}\end{array}$ & $\begin{array}{l}\text { Descritivo } \\
\text { Transversal }\end{array}$ & $\begin{array}{l}\text { Acta Paulista de } \\
\text { Enfermagem }\end{array}$ & 70 enfermeiros & $\begin{array}{l}\text { Consulta de enfermagem, reuniões educati- } \\
\text { vas, investigação do risco, ECM, orientações } \\
\text { AEM, solicitação de MMG. }\end{array}$ \\
\hline
\end{tabular}


A7

OLIVEIRA; MILANEZ;

VILARINHO, 2018

FERRARI et al., $2018^{17}$

A8

A9

BELFORT et al., $2019^{18}$

A10

SILVA et al., $2019^{19}$
Descritivo

Transversal

Quantitativo

Descritivo

Qualitativo

Descritivo

Qualitativo

Research, Society

and Development

Revista de enfermagem da UFPE

10 enfermeiros

Revista de Enfermagem da UFPI

80 usuárias

10 enfermeiros

Epidemiologia e

Serviços de Saúde

400 usuárias
ECM, consulta de enfermagem, solicitação de MMG E investigação do risco.

Orientações, AEM, ECM, solicitação de $M M G$, estimulo ao autocuidado.

Educação em saúde, consulta de enfermagem, ECM, encaminhamento, promoção do autocuidado, apoio emocional e acolhimento.

Solicitação de MMG, estimulo ao autocuidado, busca ativa.

Fonte: Dados da pesquisa

Os resultados apresentados demonstram que houve uma variação do número de artigos produzidos em função do ano. A análise revela que o ano de 2017 apresentou maior número de publicações, 3 (30\%). Seis artigos foram publicados em 2015 ( $n=2 ; 20 \%), 2018$ $(\mathrm{n}=2 ; 20 \%)$ e $2019(\mathrm{n}=2 ; 20 \%)$. Foi selecionado um estudo em $2016(n=1$; $10 \%)$. Não houve publicações relevantes em 2020 (Figura 1).

\section{DISCUSSÃO}

As publicações acerca das ações do enfermeiro no rastreamento e detecção precoce do câncer de mama na ESF foram escassas. Em todos os estudos foi ressalta- do o potencial do enfermeiro na realização de ações educativas na capacitação das mulheres para realização do Auto Exame da Mama (AEM), agendamento do retorno às consultas e encaminhamentos aos serviços especializados. Entretanto, não houve descrição quanto à execução do exame clínico das mamas em todas as consultas obedecendo, sobretudo todos os passos propedêuticos.

A maioria dos artigos descreveram como ações primordiais no rastreamento a consulta de enfermagem, Exame Clínico da Mama (ECM), e ações educativas. No ECM, três artigos exploraram sua periodicidade, indicação e o momento em que os enfermeiros o realizam na consulta ou em atividades educativas ${ }^{16,17,18}$.

\section{Figura 1-Distribuição dos artigos, conforme ano de publicação 2015-2020}

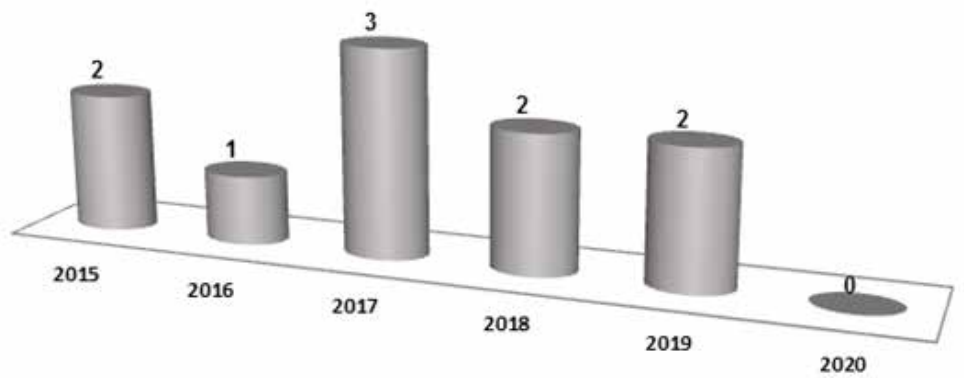

Eive de artigos

Fonte: Dados da pesquisa.

Quatro artigos ressaltaram como principais fatores que interferem nas ações do enfermeiro no processo de rastreamento e detecção precoce do câncer de mama são a estrutura física inadequada para atividades educativas, sobrecarga de atribuições, demanda excessiva de atendimentos e rotatividade de profissionais na unidade básica de saúde que impossibilita a criação de vínculo $^{12,14,16,19}$.

Desse modo, percebe-se nos estudos, que o enfermeiro atua desde a busca das mulheres, rastreamento e diagnóstico, até a implementação e a utilização dos sistemas de informação nas unidades básicas para monitoramento e controle da patologia que gera subsídio para o planejamento de ações ${ }^{13}$. Nesse sentido, um estudo realizado em 2017, com 70 enfermeiros, identificou que os fatores que interferem na qualidade das ações de rastreamento é o excesso de atribuições. Assim, acredita-se que com a capacitação, por meio da educação permanente, os profissionais terão maior subsídio de desenvolver atividades na atenção básica ${ }^{16}$.

Assim, identificou-se o papel fundamental do enfermeiro no rastreamento do câncer de mama durante as consultas. $\mathrm{Na}$ oportunidade, além dos procedimentos, devem-se utilizar estratégias educativas para sensibilizar as mulheres e aumentar a adesão, gerando a consciência do autocuidado preconizado na ESF. 


\section{artigo}

Dantas Silva, R.R.D.; Santos, T.S.; Ramos, W.T.; Barreiro, M.S.C.; Mendes, R.B.; Freitas, C.K.A.C.

Ações do enfermeiro para prevenção e detecção precoce do câncer de mama

\section{CONCLUSÃO}

O enfermeiro é fundamental para prevenção e detecção precoce do câncer de mama, seja através da realização das consultas de enfermagem, exame clínico ou solicitação de exames. Entretanto, foi constatado, a partir dessa revisão, uma escassez de estudos que abordem a temática frente à política de prevenção dessa doença no país.

Ainda, alguns estudos ressaltaram o baixo nível de conhecimento dos enfermeiros acerca da possibilidade de requisitar mamografia. Situação que seria evitada,

\section{O enfermeiro é}

fundamental para prevenção e detecção precoce do câncer de mama... mediante a instituição de protocolos bem definidos que reorientem seu trabalho na ESF, além de ampliar as competências desses profissionais.

Diante disso, compreende-se que as ações do enfermeiro para prevenção e detecção precoce do câncer de mama ainda são incipientes, devido ao pouco conhecimento técnico-científico e a ausência de capacitação. Assim, evidencia-se a necessidade da instituição de educação permanente pelos gestores, bem como a de protocolos que ampliem a autonomia dos profissionais. -

\section{REFERÊNCIAS}

1. Brasil. Ministério da Saúde. Instituto Nacional de Câncer José Alencar Gomes da Silva. Estimativas 2016: incidência de câncer no Brasil. Rio de Janeiro: Inca; 2016.

2. Souza, G. R. M. D., Cazola, L. H. D. O., \& Pícoli, R. P. (2018). Atuação do enfermeiro da atenção primária à saúde na assistência oncológica: revisão integrativa. Cogitare enferm, e58152-e58152.

3. Marques CAV, Figueiredo EM, Gutiérrez MGR. Políticas de saúde pública para o controle do câncer de mama no Brasil. Rev Enferm UERJ. 2015; 23 (2): 272-8.

4. Brasil. Decreto n 9.795, de 17 de maio de 2019. Diário Oficial da União 2019; 20 maio.

5. Brasil. Ministério da Saúde. Instituto Nacional de Câncer José Alencar Gomes da Silva. Estimativas 2020: incidência de câncer no Brasil. Rio de Janeiro: Inca; 2019.

6. Gonçalves, C. V., Camargo, V. P., Cagol, J. M., Miranda, B., \& Mendoza-Sassi, R. A. (2017). O conhecimento de mulheres sobre os métodos para prevenção secundária do câncer de mama. Ciência \& Saúde Coletiva, 22, 4073-4082.

7. Bushatsky, M., Barros, M. B. S. C., da Rocha Cabral, L., da Rocha Cabral, J., da Silva Bezerra, J. R., \& dos Santos Figueira Filho, A. S. (2014). Câncer de mama: ações de prevenção na estratégia de saúde da família. Revista de Pesquisa Cuidado é Fundamental Online, 6(2), 663-675.

8. Nogueira, I. S., Previato, G. F., Baldissera, V. D. A., Paiano, M., \& Salci, M. A. (2019). Atuação do enfermeiro na atenção primária à saúde na temática do câncer: do real ao ideal. Revista de Pesquisa: Cuidado é Fundamental, 725-731.

9. Mendes KDS, Silveira RCCP, Galvão CM. Revisão integrativa: método de pesquisa para a incorporação de evidências na saúde e na enfermagem. Texto Contexto Enferm. 2008;17(4):758-64

10. Duarte, S. J. H., Gaspar, R. A., Alves, V. H., \& Rodrigues, D. P. (2015). Prevenção do câncer de mamas e colo uterino na perspectiva de mulheres: implicações para o serviço. Revista de Enfermagem do Centro-Oeste Mineiro, 5(1).

11. Zapponi, A. L. B., Tocantins, F. R., \& Vargens, O. M. D. C. (2015).
O enfermeiro na detecção precoce do câncer de mama no âmbito da atenção primária. Rev. enferm. UERJ, 23(1), 33-38.

12. de Moraes, D. C., de Almeida, A. M., de Figueiredo, E. N., de Loyola, E. A. C., \& Panobianco, M. S. (2016). Rastreamento oportunístico do câncer de mama desenvolvido por enfermeiros da Atenção Primária à Saúde. Revista da Escola de Enfermagem da USP, 50(1), 14-21.

13. Marques, C. A. V., da Silva, V. R., \& Gutiérrez, M. G. R. (2017). Ações do enfermeiro na detecção precoce do câncer mamário [Nurses' role in early detection of breast cancer][Acciones del enfermero en la detección temprana del cáncer de mama]. Revista Enfermagem UERJ, 25, 22639.

14. Melo, F. B. B., Marques, C. A. V., Rosa, A. D. S., Figueiredo, E. N. D., \& Gutiérrez, M. G. R. D. (2017). Ações do enfermeiro na detecção precoce do câncer de mama. Revista Brasileira de Enfermagem, 70(6), 1119-1128.

15. Teixeira, M. S., Goldman, R. E., Gonçalves, V. C. S., de Gutiérrez, M. G. R., \& de Figueiredo, E. N. (2017). Atuação do enfermeiro da Atenção Primária no controle do câncer de mama. Acta Paulista de Enfermagem, 30(1), 1-7.

16. Oliveira, V. A. D. S., Vilarinho, M. L. C. M., \& Milanez, L. D. S. (2018). Caracterização de mulheres com risco do câncer de mama na Atenção Primária à Saúde. Rev. enferm. UFPI, 38-43.

17. Ferrari, C. F., Abreu, E. C. D., Trigueiro, T. H., Silva, M. B. G. M. D., Kochla, K. A., \& Souza, S. R. R. K. (2018). Orientações de cuidado do enfermeiro para a mulher em tratamento para câncer de mama. Rev. enferm. UFPE on line, 676-683.

18. Belfort, L. R. M., Magalhães, K., Dutra, L. P. F., Negro Dellacqua, M., da Silva Martins, V. H., \& de Andrade Macedo, L. J. (2019). 0 papel do enfermeiro no diagnóstico precoce do câncer de mama na atenção primária. Research, Society and Development, 8(5), 12.

19. Silva, R. D. P., Gigante, D. P., Amorim, M. H. C., \& Leite, F. M. C. (2019). Fatores associados à realização de mamografia em usuárias da atenção primária à saúde em Vitória, Espírito Santo. Epidemiologia e Serviços de Saúde, Brasília, 28(1), 1-11. 